\title{
Reduction of Matrix Metalloprotease 3 in Rheumatoid Arthritis Rat by Low Level Diode Laser Irradiation
}

\author{
Masaaki Shimada, ${ }^{1}$ Noboru Kuboyama, ${ }^{2}$ and Yoshimitsu Abiko ${ }^{1}$
}

Departments of ${ }^{1}$ Biochemistry and Molecular Biology, and ${ }^{2}$ Molecular Pharmacology, Nihon University School of Dentistry at Matsudo, Matsudo, Chiba 271-8587, Japan

Correspondence to :

Yoshimitsu Abiko

E-mail : abiko.yoshimitsu@ nihon-u. ac.jp

\section{Keywords :}

rheumatoid arthritis, rat joint, matrix metalloprotease 3, low-level laser irradiation

\begin{abstract}
Rheumatoid arthritis (RA) is an inflammatory joint disorder, whose progression leads to the destruction of cartilage and bone. Low-level laser irradiation (LLLI) is currently being evaluated for the treatment of RA; however, the molecular mechanisms underlying its effectiveness remain unclear. To understand the anti-inflammatory effect of LLLI, we used the collagen-induced RA rat (CIA) model, and analyzed the gene expression profile in synovial membrane in knee joints. Total RNA was isolated from the synovial membrane tissue of CIA rat joints or CIA joints treated with LLLI (830 nm Ga-Al-As diode laser), and gene expression profiles were analyzed by DNA microarray (41,000 rat genes) and Ingenuity Pathway Analysis system (IPA). The mRNA levels were confirmed by reverse transcription polymerase chain reaction (RT-PCR) and real-time PCR. Proteins expression was also carried out by immunohistochemistry. DNA microarray and IPA network/canonical pathway analysis showed that matrix metalloproteases (MMPs) signaling was involved, among MMPs, MMP3 gene expression was increased in CIA tissue, and LLLI significantly decreased the gene expressio. The DNA microarray data of these mRNA levels were further validated and confirmed using RT-PCR and realtime PCR. Immunohistochemical study also demonstrated the reduction of MMP2 protein level by LLLI. Since MMP3 These findings suggest that inhibitory effect of MMP3 expression may be a part of decreased CXCL1 and ICAM1-1 expressions may be a part of the mechanisms in LLLI-mediated reduction of RA inflammation.
\end{abstract}

\section{Introduction}

Rheumatoid arthritis (RA) is a chronic inflammatory disease characterized by pain and inflammation, progressive joint destruction, significant disability, systemic manifestations and premature mortality (1). This disease is characterized by the infiltration of leukocytes into the synovial tissue and synovial fluid of joints, ultimately leading to destruction of cartilage and bone (2). Inflammation of the synovial lining of joints results in further increase in the expression of inflammatory cytokines and chemokine-mediated recruitment of additional inflammatory cells, as well as activation of $\mathrm{B}$ cells with autoantibody production. A vicious cycle of altered cytokine and signal transduction pathways and inhibition of programmed cell death contribute to cartilage and bone destruction by human fibroblast-like synoviocytes and osteoclasts $(3-5)$.

Collagen-induced arthritis (CIA) is an animal model of RA that is widely used to address questions regarding the pathogenesis of $\mathrm{RA}$ and to validate therapeutic targets. The pathological features of CIA include proliferative synovitis with infiltration of polymorphonuclear and mononuclear cells, pannus formation, cartilage degradation, and erosion of bone (6).

A non-invasive physiological therapy such as low level laser irradiation (LLLI) could be important for 
managing pain. Since the early 1960s with different wavelength laser therapies have been used in the duration of acute inflammation, the stimulation of tissue repair and the relief from pain, and bone formation (7). Different wavelength of laser including $\mathrm{Ga}^{-} \mathrm{Al}-\mathrm{As}$ diode laser. However, there are few studies on the mechanism of anti-inflammatory effects; most of the studies deal only with the analgesic properties of laser and the efficacy of LLLI as an anti-inflammatory therapy is still controversial (8). It is necessary to analyze simultaneous expression levels of thousands of genes. In this context, a powerful way to gain insight into the molecular complexity and pathogenesis of arthritis has arisen from oligonucleotide-based microarray technology, because this platform provides an opportunity to analyze simultaneously the expression of a large number of genes in disease tissues.

In the present study, whole homogenized rat knee joints synovium from normal, CIA and CIA with LLLI rats were analyzed for differential gene expression using the microarray. Furthermore, the genes identified were analyzed for network by ingenuity pathway analysis (IPA) software to identify networks of interacting genes.

\section{Materials and Methods}

\section{Animal experiment}

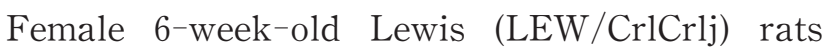
were obtained from Charles River Japan Inc. (Kanagawa, Japan). The rats were allowed free access to food and water available ad libitum at all times and were maintained in $12 \mathrm{~h}$ light/dark cycle (lights on 6 : 00 to $18: 00)$ at $23 \pm 1{ }^{\circ} \mathrm{C}, 60 \pm 10 \%$ humidity for a period of 1 week before use. Type II collagen-induced arthritis (CIA) was induced in rats by multiple intradermal injections, at the base of the tail and into three to five other sites on the back, of $200 \mu \mathrm{g}$ of bovine type II collagen (Sigma, Tokyo, Japan) in 250 $\mu 1$ of $0.1 \mathrm{M}$ acetic acid emulsified in an equal volume of complete Freund's adjuvant (Difco Labs, Michigan, USA) containing $2 \mathrm{mg} / \mathrm{ml}$ Muramyl Dipeptide (Wako, Tokyo, Japan). 14 days later the rats were again challenged with the same antigen prepa- ration. All animals were anaesthetized with an intraperitoneal injection of sodium pentobarbital (SomnopentylR, Kyoritsu Seiyaku, Tokyo, Japan) at $25 \mathrm{mg} / \mathrm{kg}$ prior to immunization. CIA rats developed 2 weeks after the third immunization. All animals were maintained and used in accordance with the guide to the Care and Use of Laboratory Animals of Nihon University, School of Dentistry at Matsudo (No. 04-008).

\section{LLLI experiment}

A Ga-Al-As diode laser device (Model ZH-M143 DJP; Panasonic Industrial Equipment Inc., Japan) was used as a low-power laser source. This laser therapy system utilizes a wavelength of $830 \mathrm{~nm}$ and output power of $100-700 \mathrm{~mW}$, which is variable in a continuous wave $(\mathrm{c} / \mathrm{w})$. In the present study, an output power in a c/w of $500 \mathrm{~mW}$ was selected. The probe was fixed at $550 \mathrm{~mm}$ from the hind-paw to be irradiated, giving a spot size of $78.5 \mathrm{~cm}^{2}$, with an incident power density of approximately $6.4 \mathrm{~mW} /$ $\mathrm{cm}^{2}$. The irradiation time was $20 \mathrm{~min}$, which was equivalent to an incident energy density of $7.64 \mathrm{~J} /$ $\mathrm{cm}^{2}$. LLI experiment was carried out three times a week for a period of 2 weeks.

\section{DNA microarray analysis}

Total cellular RNA was isolated from the knee joint synovial membrane using RNeasy Fibrous Tissue Midi Kit Isolation System (Qiagen Ltd.) by a FastPrep machine (FP120; BIO 101). The cDNA synthesis was performed using a GeneAmp RNA PCR Kit (Applied Biosystems, Foster City, Calif.) with samples of total RNA. Labeled cRNA synthesis was used Quick Amp Labeling Kit (Agilent, Santa Clara, CA) according to the manufacturer's protocol. For hybridization, $1.65 \mu \mathrm{g}$ of cy3-labeled cRNA from treated and vehicle-treated cells was combined and hybridized to a Whole Rat Genome Microarray $4 \times 44$ K (41,000 rat genes and transcripts ; Agilent) according to the manufacturer's protocol. Finally, the arrays were scanned using an Agilent Microarray Scanner (Agilent), and the scanned images were captured using Agilent Feature Extraction software 
(Agilent) and analyzed using GeneSpring GX10 software (Agilent Technologies, Inc Santa Clara, CA).

\section{Ingenuity Pathways Analysis (IPA)}

To facilitate the analysis of the gene microarray data, and to relate gene up- and down-regulation to underlying biological processes, Ingenuity Pathways Analysis (IPA) (Ingenuity ${ }^{\circledR}$ Systems, www.ingenuity. com) has proposed using in silico genomics network analysis [8]. IPA version 4.0 (Ingenuity Systems, Mountain View, CA, USA) was used to search for possible biological processes, pathways and networks. This web-based entry tool allows for the mapping of gene expression data into relevant pathways based on their functional annotation and known molecular interactions [9]. This knowledge coming from published, peer-reviewed scientific publications is stored in the Ingenuity Pathways Knowledge Base (IPKB), and is continuously updated. A molecular network of direct or indirect physical, transcriptional, and enzymatic interactions between mammalian orthologs was computed from this knowledge base. By comparing the imported microarray data with the IPKB, the list of genes was transformed in a set of relevant networks, focus genes and canonical pathways were identified, and functional annotation was performed.

Focus genes identified by the IPA program were categorized based on location, cellular components, and reported biochemical, biologic, and molecular functions using the software. The identified genes also were mapped to genetic networks in the IPA database and ranked by score. The score reflects the probability that a collection of genes equal to or greater than the number in a network could be achieved by chance alone. Canonical pathways analysis identified molecular pathways from the IPA library of canonical pathways (part of the IPKB) that were most significant to the data set. Genes from the data set that were associated with a canonical pathway in the IPKB were considered for the analysis.

\section{RT-PCR and real-time PCR Analysis}

$\mathrm{RT}$-PCR and real-time PCR reactions were carried out using a DNA thermal analyzer (RFNGene $^{\mathrm{TM}} 6000$; Corbett Life Science, Sidney, Australia). Amplification by PCR was started with an initial incubation at $95^{\circ} \mathrm{C}$ for 15 seconds to activate the Taq DNA polymerase, and then performed at 95 ${ }^{\circ} \mathrm{C}$ for 5 seconds and $56{ }^{\circ} \mathrm{C}$ for 15 seconds by adequate cycles. RT-PCR products were electrophoresed on $1.5 \%$ agarose gel, followed by staining with ethidium bromide to examine the size of the PCR products. Real-time PCR was carried out with SYBR Premix Ex Taq ${ }^{\text {TM }}$ (Perfect Real-Time PCR, Takara, Japan). To calculate gene expression fold changes, the initial template concentration was derived from the cycle number at which the fluorescent signal crossed the threshold in the exponential phase of the real-time PCR reaction. The mRNA copy unit was given by the cycle threshold value from the fluorescent signal of all the samples, including the standard curve and target genes, following the method provided by Corbett Life Science Company using RFN-Gene ${ }^{\mathrm{TM}}$ 6000 software. Details are described in the operation manual, version 1.7.40, 2006. Each assay was normalized to GAPDH (glyceraldehyde-3-phosphate dehydrogenase) mRNA levels.

The DNA primer sequences were ; 5'-gctcatcctacc cattgcat-3' (the forward primer for MMP3 gene) ; 5' -gcttgtgcatcagctccata-3' (the reverse primer for MMP3 gene), (predicted size $=219 \mathrm{bp}) ; 5$ '-atcaccatct tccaggag-3' (the forward primer for gapdh) ; and 5'atcgactgtggtcatgag-3' (the reverse primer for GAPDH gene), (predicted size $=318 \mathrm{bp}$ ). Values were calculated as means \pm standard deviation (SD). Comparisons were made between two groups using a Student's t-test.

\section{Immunhistochemistry}

The hindpaws of each rat were dissected and fixed immediately by immersion in 10\% buffered formalin for $6 \mathrm{hrs}$ and decalcified with 10\% tetrasodiumEDTA aqueous solution ( $\mathrm{pH}$ 7.4) for 4 weeks at room temperature. The decalcifying solution was changed twice each week. Tissue specimens were processed 
for paraffin wax embedding and multiple $4 \mu \mathrm{m}$ sections were prepared form each block for immunohistochemical examinations. For immunohistochemistry analysis serial sections were treated in a microwave oven four times with citrate buffer ( $\mathrm{pH}$ 6.0) for $5 \mathrm{~min}$ at $960 \mathrm{~W}$. Sections were saturated with $10 \%$ BSA in PBS with $0.1 \%$ Triton $\mathrm{X}-100$ and incubated overnight at $4{ }^{\circ} \mathrm{C}$ in a humidified chamber with the anti-MMP3 rabbit polyclonal antibody (LifeSpan Bioscience, Inc., WA, USA). Subsequent incubations were made with biotinylated goat anti rabbit IgG $(\mathrm{H}+\mathrm{L})$ (Vector) followed by horseradish peroxidase streptavidin (Vector) for $1 \mathrm{hr}$ at room temperature.
The color was developed with 3-3'-diamino benzidine tetrahydrochloride. Sections were counterstained with Mayer's hematoxylin.

\section{Results}

Gene expression profiles of control, CIA and CIA + LLLI rat joint synovial membrane tissues were analyzed using a high-throughput DNA microarray. Data mining was carried out by cut-off values of $\geq 2.0$ for "induction" and $\leq 0.5$ for "repression". To further refine the significance of the altered gene identified in DNA microarray data, we investigated possible biological interactions using the IPA tool. We asked

\section{(A) Molecules in net work (CIA vs control)}

\begin{tabular}{|c|c|}
\hline & Molecules in Network \\
\hline & 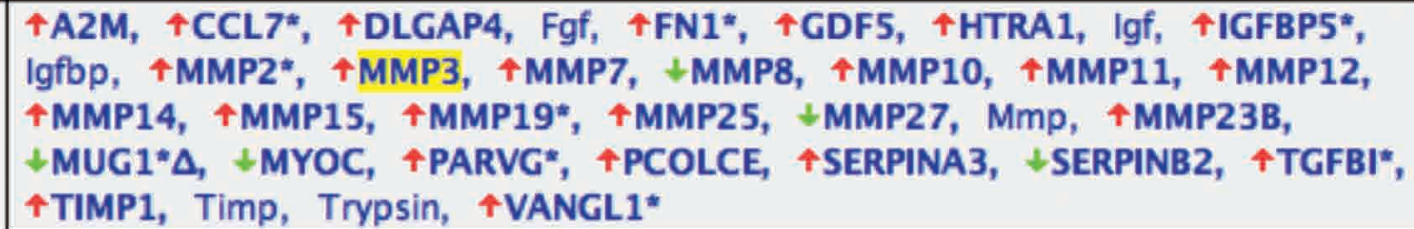 \\
\hline
\end{tabular}

\section{(B) Network searchof MMP family (CIA vs control)}

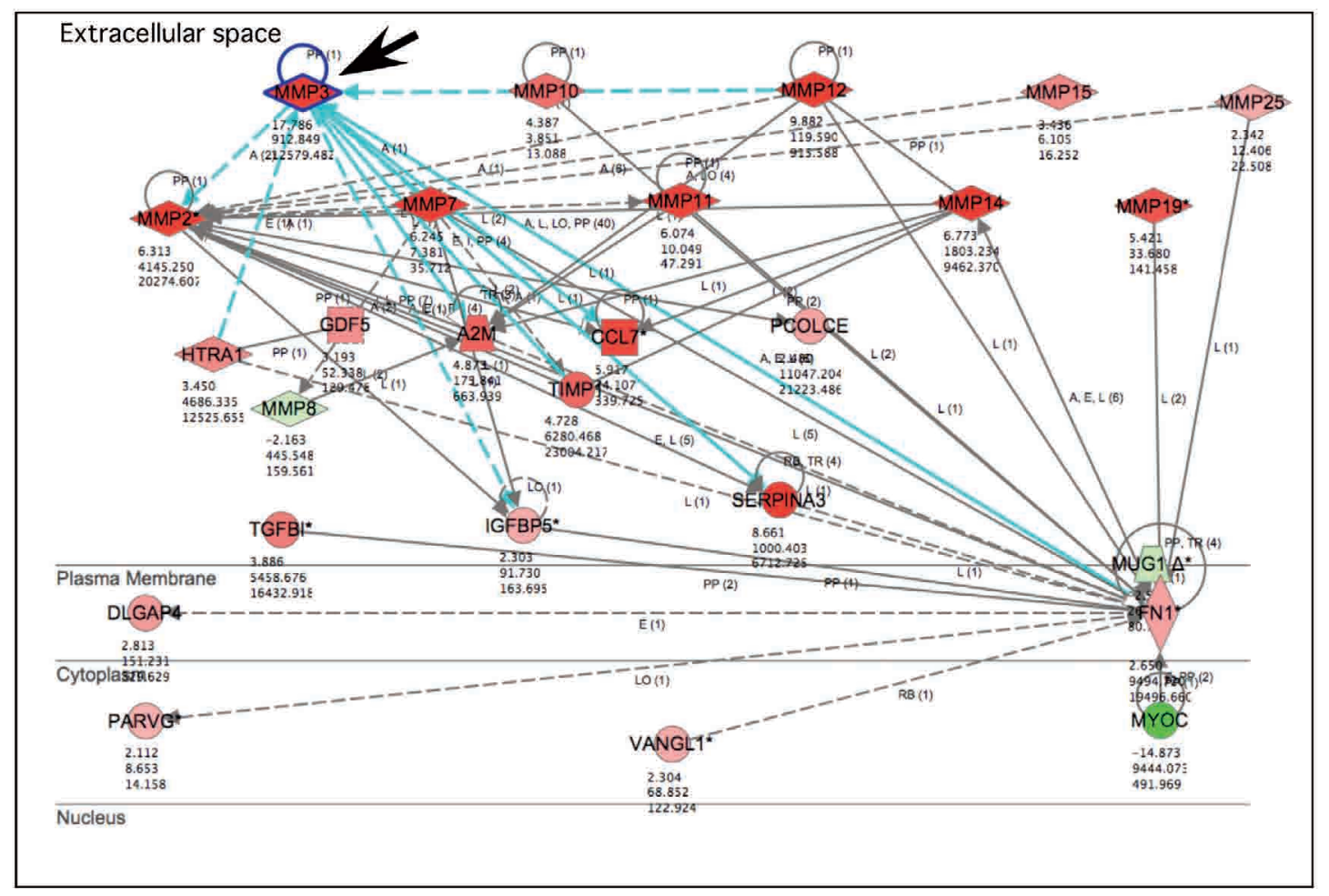

Fig. 1. Network search of CIA vs control using IPA sytem. (A) Molecules in the network from CIA vs control using MMP3 as a landmark (marked by yellow). (B) The relation of molecules in the network (CIA vs control). Arrow indicates MMP3 gene. 
the network search in the IPA database system using MMP3 as a landmark, and identified a network which included many MMP gene families in network molecules (Fig. 1A). The CIA vs control networks indicated functional relationships between gene products based on known interactions reported in the literature including MMPs. The relation of molecules in networks (CIA vs control) are illustrated Fig. 1B. The MMP3 gene expression was highly upregulated (17.7-fold) in CIA rat when compared with

\section{(A) Molecules in network (CIA + LLLI vs CIA)}

\begin{tabular}{|c|}
\hline Molecules in Network \\
\hline 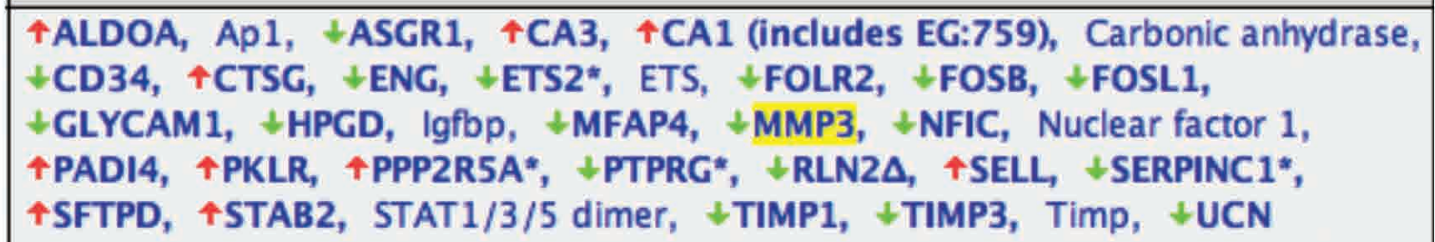 \\
\hline
\end{tabular}

(B) Network search of MMP family (CIA + LLLI vs control)

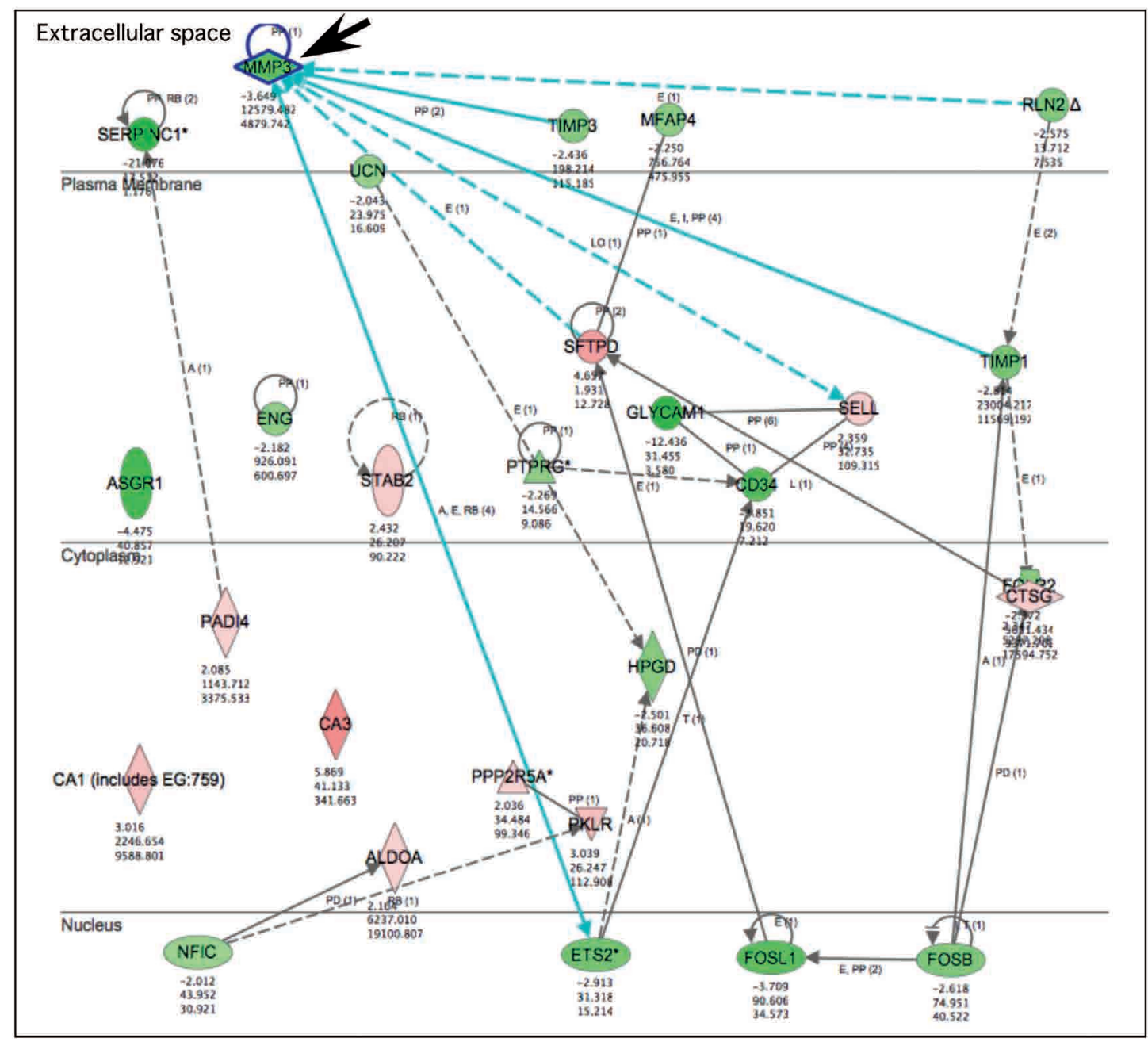

Fig. 2. Network search of CIA + LLLI vs CIA using IPA sytem. (A) Molecules in the network from CIA + LLLI vs CIA using MMP3 as a landmark (marked by yellow). (B) The relation of molecules in networks (CIA+LLLI vs CIA). Arrow indicates MMP3 gene. 
control.

Next we searched a network of effect of LLLI on CIA (CIA + LLLI vs CIA). As we expected, the MMP3 gene expression was down-regulated in LLLI rats when compared with CIA rats (Fig. 2AB).

Gene expression profiles of (CIA vs control) and (CIA+LLLI vs CIA) were summarized as the scatter plots shown in Fig. 3.

The IPA system is not only able to produce a gene network map but also can facilitate the elucidation of canonical pathway analysis. Relationships between networks generated by the IPA system and known pathways were further investigated by IPA canonical pathway analysis. We focused on "Role of Macrophages, Fibroblasts and Endothelial cells in Rheumatoid Arthritis" cytokine signaling pathway. As shown in Fig. 4, CIA highly increased gene expression of MMP3 gene (17.7-fold) with TNFR, IL-1 and IL-1R and $\mathrm{c}^{-}$jun genes, but not MMP1 and MMP13 genes. Interestingly, LLLI suppressed the signaling by decreasing IL-1R and MMP3 gene expression (3.6 -fold) by LLLI in CIA rat joint tissues.

In order to validate the results obtained from IPA, we examined the MMP3 gene expressionby RTPCR. As shown in Fig. 5, an amplified DNA band corresponding to MMP3 gene in CIA rats was more intense than in the control rats, whereas LLLI reduced the intensity of this DNA band. In contrast, mRNA levels of GAPDH, a housekeeping control, were not different between the CIA rats and LLLItreated CIA rats.

To determine the exact rates of enhanced expression for MMP3 gene, we performed real-time PCR. The real-time PCR results were converted to mRNA relative copy units were calculated. As shown in Fig. 6, there was an increase in expression of MMP3 gene in CIA rats, and a significant decrease in expression following LLLI.

Finally, phenotypic expression of MMP3 proteins was examined by immuno-histochemistry. MMP3 proteins could be detected in the synovial tissues of all groups of rats. The synovial tissue from control rat exhibited very weak constitutive expression of MMP3 protein, and was strongly up-regulated in
CIA rats. As we expected. MMP3 protein expression was significantly decreased in CIA rats by LLLI. Staining was negative when the primary antibody was omitted (Fig. 7).
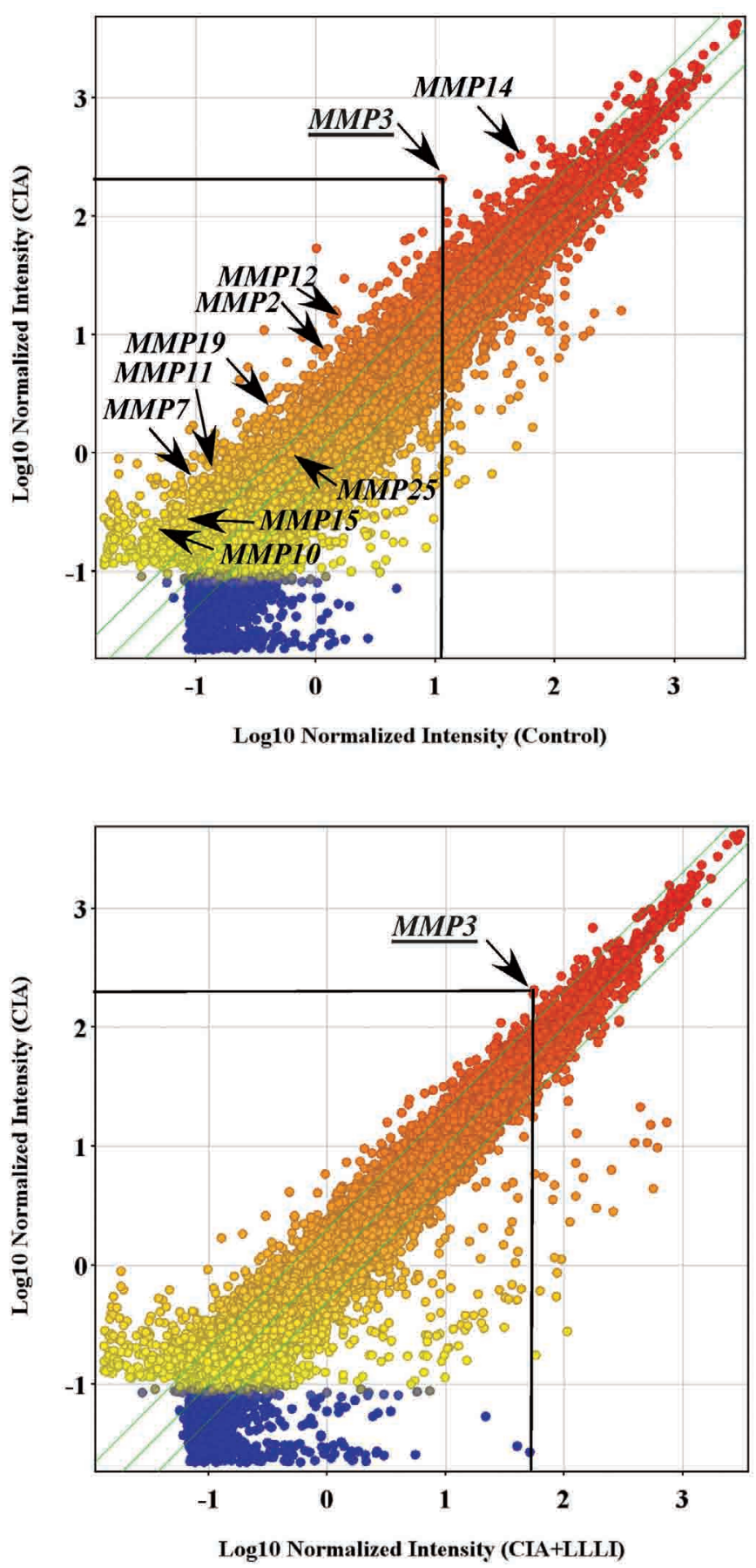

Fig. 3. Canonical pathway analysis of "Role of Macrophages, Fibroblasts and Endothelial cells in Rheumatoid Arthritis". Red, up-regulated; green, down-regulated. First line under mark is fold change, second line is raw intensity of CIA, and third line is raw intensity of CIA + LLLI. 


\section{CIA vs control}

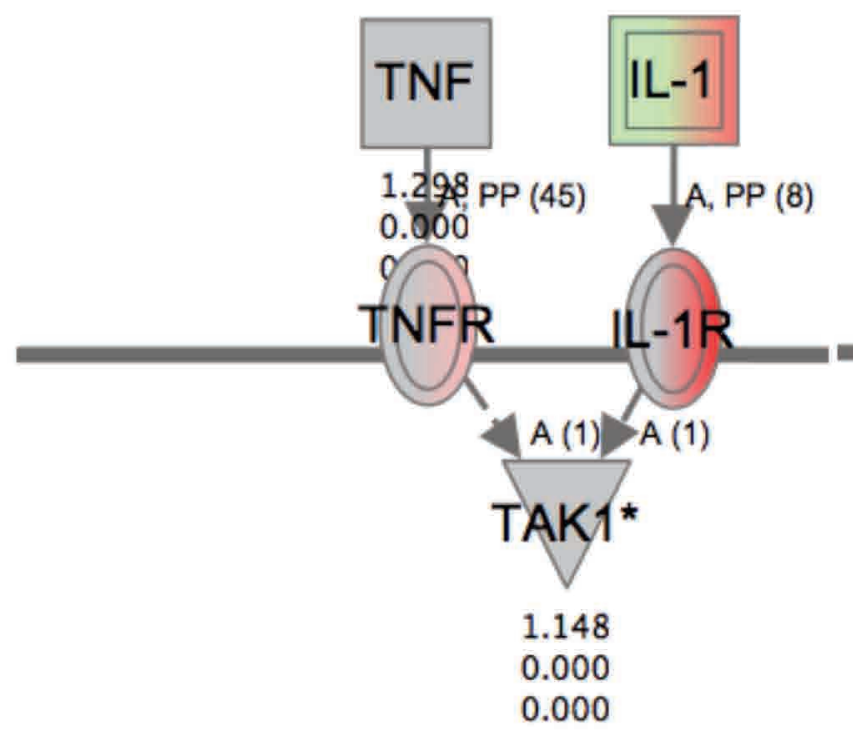

CIA+ LLLI vs CIA

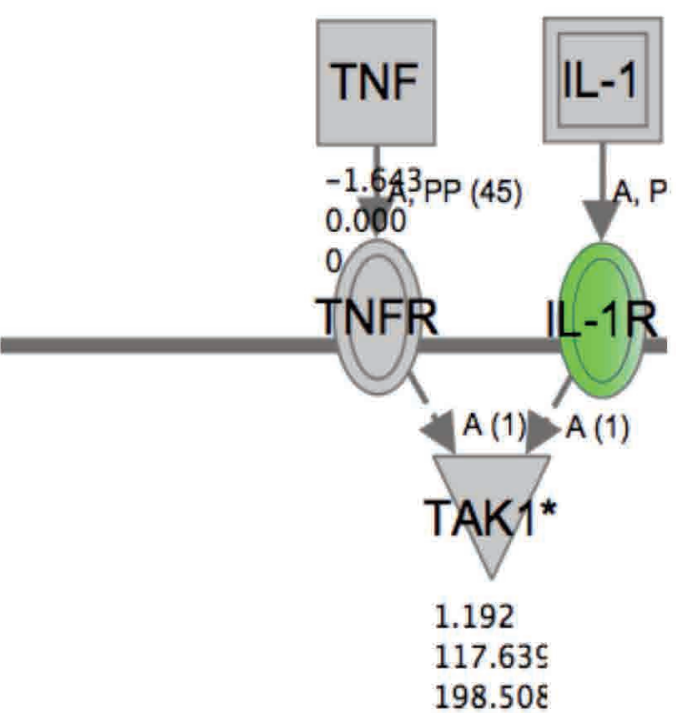

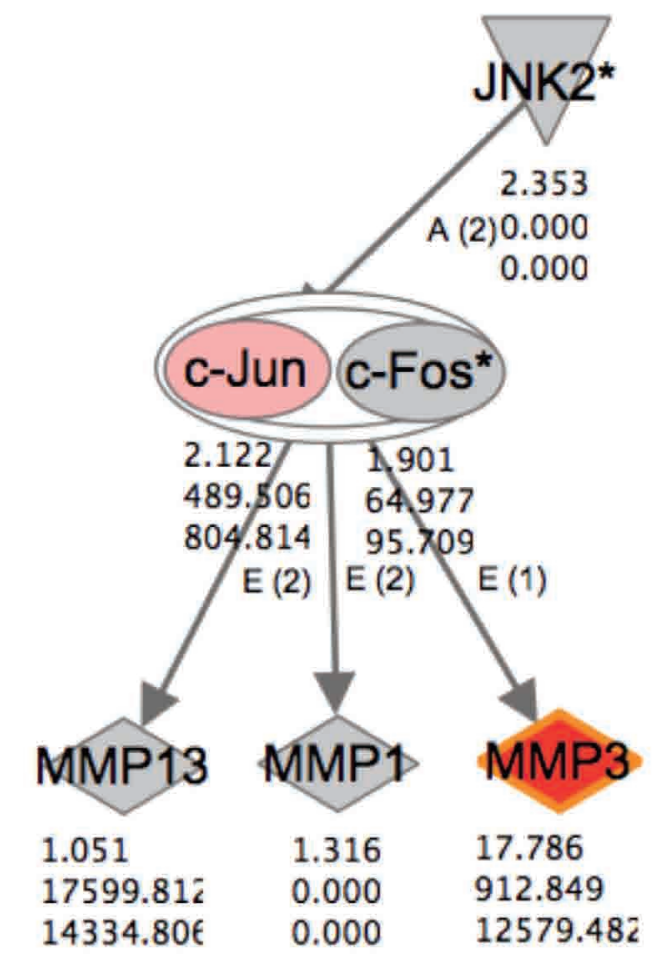

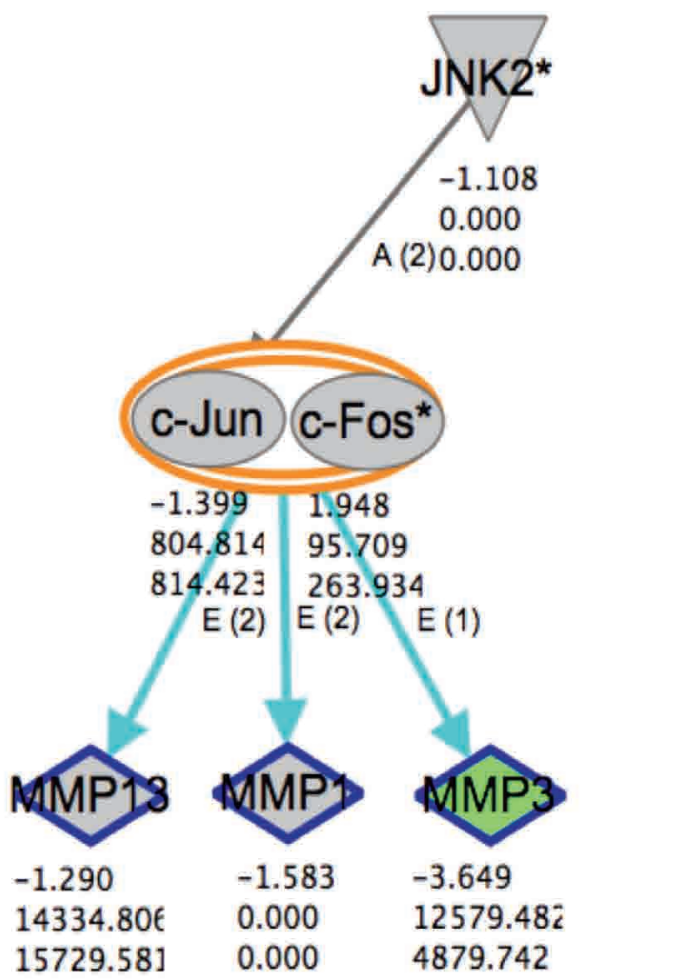

Fig. 4. Scatter plot of gene expression of MMP gene families. Left, CIA vs control ; right, CIA+LLLI vs CIA.

\section{Discussion}

$\mathrm{RA}$ is a persistent, systemic inflammatory disease, characterized by inflammation involving multiple cell types, resulting in the progressive destruction of the affected joints. Among dozens of MMPs, MMP3 has been reported to be the major enzyme produced by fibroblasts and macrophage-like cells in the synovium, and the level of MMP3 has been reported to be significantly higher in synovial fluids from patients with RA (9-11). Destruction of cartilage is a pathological feature in RA, and is a major cause of joint dysfunction in RA patients. Many proteinases 


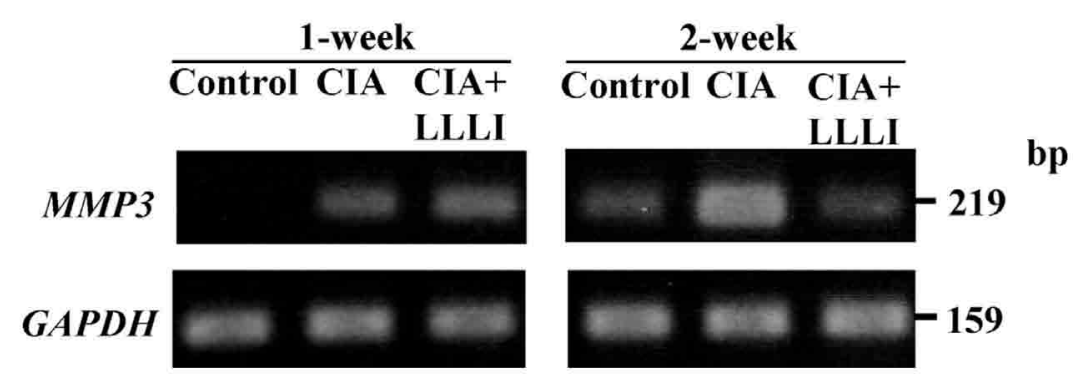

Fig. 5. RT-PCR analysis of mRNA levels of MMP3 gene. An ethidium bromide-staining pattern of the amplified PCR products using agarose gel electrophoresis.

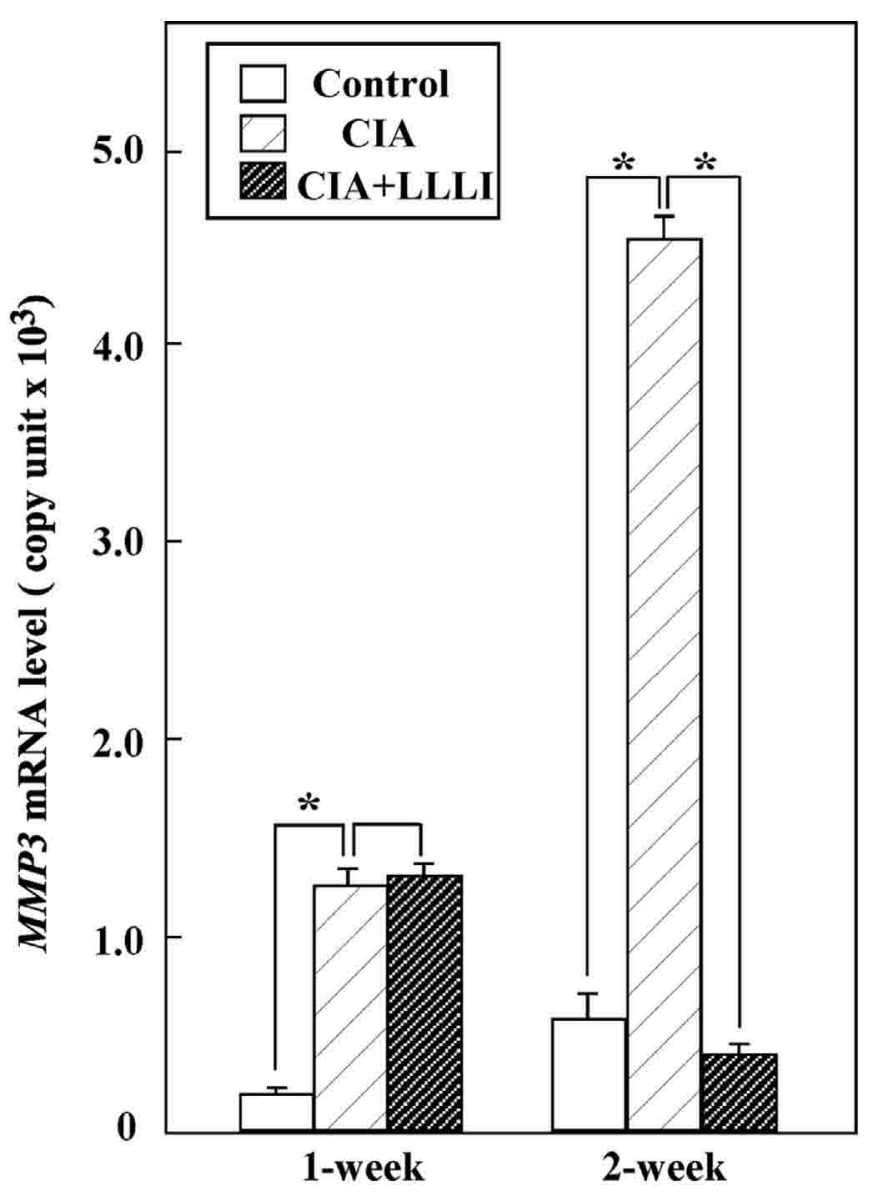

Fig. 6. Real-time PCR analysis of MMP3 gene mRNA levels. Results were expressed as relative mRNA copy unit by normalization to a housekeeping gene (GAPDH). ${ }^{*} \mathrm{p}<0.01, \mathrm{n}=4$.

are expressed in RA joint tissues of RA patients. MMPs are believed to have a key role in the joint destruction in the arthritides (12).

MMPs, a gene family of neutral $\mathrm{Zn}^{2+}$ dependent metalloproteinases, which are classified into five subgroups: (1) collagenases including tissue collagenase (MMP1), neutrophil collagenase (MMP8),

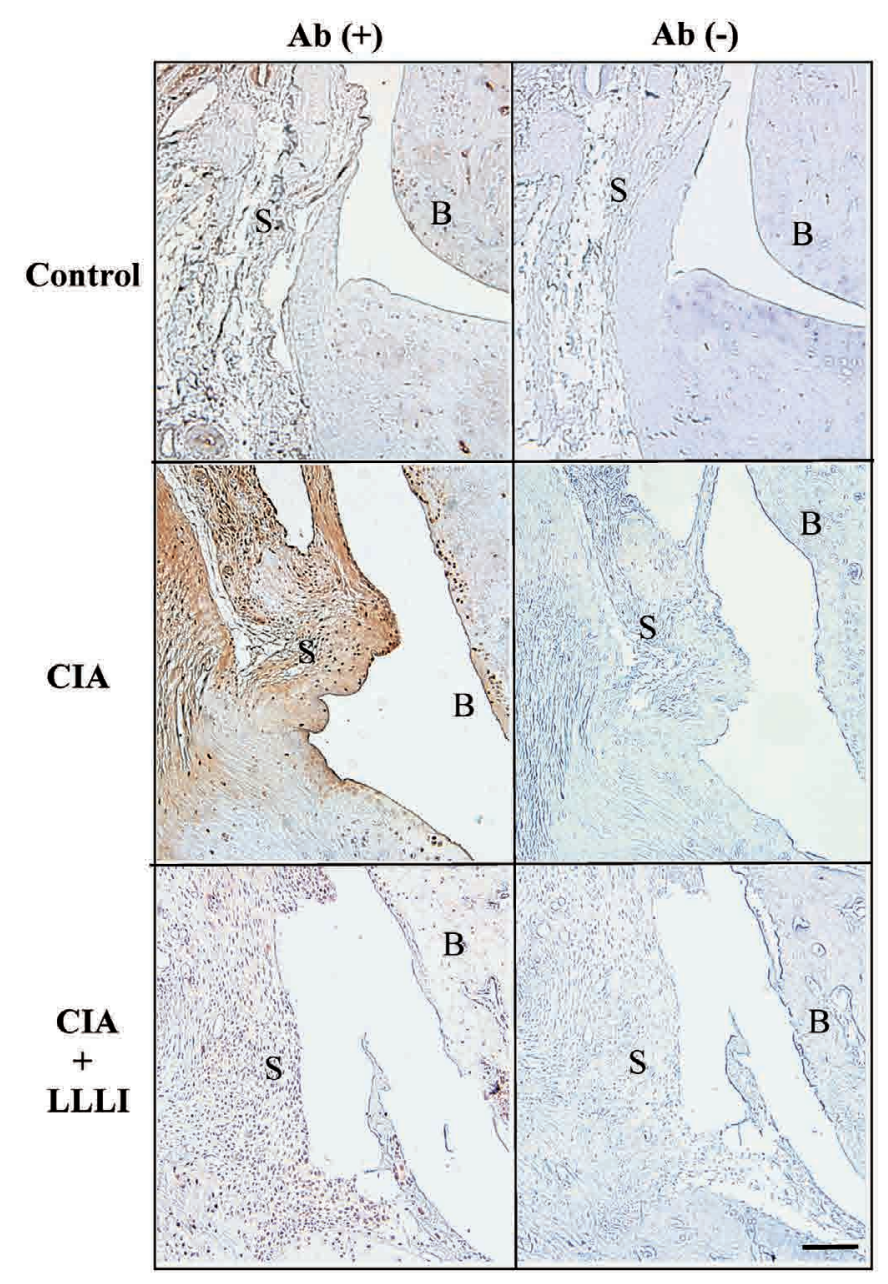

Fig. 7. Immunohistochemical study of MMP3 protein expression in rat synovial tissue. Ab, antibody against MMP3; S, synovial membrane; $\mathrm{B}$, bone. Bar $=100 \mu \mathrm{m}$.

and collagenase 3 (MMP13); (2) gelatinases including gelatinase A (MMP2) and gelatinase B (MMP9) ; (3) stromelysins including stromelysin 1 (MMP3) and stromelysin 2 (MMP10); (4) membrane-type MMPs including MT1-MMP (MMP14), MT2-MMP 
(MMP15), MT3-MMP (MMP16), MT4-MMP (MMP17), and MT5-MMP (MMP24); (5) other MMPs including matrilysin (MMP7), stromelysin 3 (MMP11), metalloelastase (MMP12), MMP19, enamelysin (MMP20), and MMP23 (13).

Our gene expression profiles between control and CIA groups using a DNA microarray demonstrated that MMP2, 3, 7, 10, 11, 12, 14, 15, 19, and 25 genes were up-regulated in CIA when compared with control (healthy normal subjects) shown in Fig. 1. Among MMP gene family, MMP1 and MMP3 are produced by synovial lining cells and can be detected in synovial fluid of RA (14), whereas MMP2 is produced by stromal cells in the sublining synovial layer (15). MMP3 is active against cartilage matrix components, such as proteoglycanand fibronectin, and can activate pro-MMPs (16). Our pathway analysis showed that MMP1 gene did not altered by LLLI, whereas only MMP3 gene expression was downregulated in CIA by LLLI (Fig. 3).

MMP3 is also known as a useful marker for prediction of joint destruction (17). It has been reported that serum MMP-3 levels correlate with MMP3 levels produced by the synovium, and thus reflect the level of activity of rheumatoid synovitis (18). In fact, the serum MMP-3 level decreases in patients who respond to anti-RA drugs (methotrexate and sulfasalazine) treatment (19).

Taken together with these investigation, inhibition of MMP3 expression in CIA synovial membrane tissues by LLLI treatment may be one of important mechanisms and may explain the beneficial effects of LLLI treatment on the RA.

\section{Acknowledgments}

This study was supported in part by the "Academic Frontier" Project for Private Universities, a matching fund subsidy from the Ministry of Education, Culture, Sports, Science and Technology, 20072011, and by Grant-in-Aid for Scientific Research from Japan Society for the Promotion of Science (B21390497). We would like to thank Dr. Lin Zhang for his excellent technical assistance.

\section{References}

1. Pincus T: Long-term outcomes in rheumatoid arthritis. Br J Rheumatol, 34 : 59-73, 1995.

2. Gay S, Gay RE, Koopman WJ : Molecular and cellular mechanisms of joint destruction in rheumatoid arthritis: two cellular mechanisms explain joint destruction? Ann Rheum Dis, 52 : 39-47, 1993.

3. Dayer JM: The saga of the discovery of $\mathrm{IL}^{-1}$ and TNF and their specific inhibitors in the pathogenesis and treatment of rheumatoid arthritis. J Bone Spine, 69 : 123-132, 2002.

4. Inoue H, Takamori M, Nagata N, Nishikawa T, Oda $\mathrm{H}$, Yamamoto S, Koshihara Y : An investigation of cell proliferation and soluble mediators induced by interleukin $1 \beta$ in human synovial fibroblasts : comparative response in osteoarthritis and rheumatoid arthritis. Inflamm Res, 50: 65-72, 2001.

5. Firestein GS: Immunologic mechanisms in the pathogenesis of rheumatoid arthritis. J Clin Rheumatol, 11: S39-S44, 2005.

6. Trentham DE: Collagen arthritis in rats, arthritogenic lymphokines and other aspects. Int Rev Immunol, 4 : 25-33, 1988.

7. Mester E, Mester AF, Mester A: The biomedical effects of laser application. Lasers Surg Med, 5 : 3139, 1985.

8. Basford JR: Low-energy laser therapy: controversies and new research findings. Lasers Surg Med, $9: 1-5,1989$.

9. Tetlow LC, Wolley DE: Comparative immuno-localization studies of collagenase- 1 and collagenase 3 production in the rheumatoid lesion, and by human chondrocytes and synoviocytes in vitro. Br J Rheumatol, 37 : 64-70, 1998.

10. Yoshihara Y, Nakamura H, Obata K, Yamada H, Hayakawa T, Fujikawa K, Okada Y : Matrix metalloproteinases and tissue inhibitors of metalloproteinases in synovial fluids from patients with rheumatoid arthritis or osteoarthritis. Ann Rheum Dis, 59 : 455-461, 2000.

11. Keyszer G, Lambiri I, Nagel R, Keysser C, Keysser M, Gromnica-Ihle E, Franz J, Burmester GR, Jung K: Circulating levels of matrix metalloproteinases MMP -3 and MMP-1, tissue inhibitor of metalloproteinases 1 (TIMP-1), and MMP-1/TIMP-1 complex in rheumatic disease. Correlation with clinical activity of rheumatoid arthritis versus other surrogate markers. J Rheumatol, 26 : 251-258, 1999.

12. Firestein GS. Etiology and pathogenesis of rheumatoid arthritis. In: Kelly WN, Harris ED Jr, Ruddy S, Sledge CB, eds. The text book of rheumatology. 5th ed. Philadelphia: Saunders, 1996: 851-897.

13. Yoshihara Y, Nakamura H, Obata K, Yamada H, Hayakawa T, Fujikawa K, Okada Y : Matrix metal- 
loproteinases and tissue inhibitors of metalloproteinases in synovial fluids from patients with rheumatoid arthritis or osteoarthritis. Ann Rheum Dis, 59: 455-461, 2000.

14. Walakovits LA, Moore VL, Bhardwaj N, Gallick GS, Lark MW : Detection of stromelysin and collagenase in synovial fluid from patients with rheumatoid arthritis and posttraumatic knee injury. Arthritis Rheum, 35: 35-42, 1992.

15. Hembry RM, Bagga MR, Reynolds JJ, Hamblen DL : Immunolocalization studies on six matrix metalloproteinases and their inhibitors, TIMP-1 and TIMP-2, in synovia from patients with osteo- and rheumatoid arthritis. Ann Rheum Dis, 54 : 25-32, 1995.

16. Nagase H : Activation mechanisms of matrix metal- loproteinases. Biol Chem, 378 : 151-160, 1997.

17. Houssiau FA, Malaise MG: Increased matrix metalloproteinase-3 serum levels in rheumatic diseases: relationship with synovitis and steroid treatment. Ann Rheum Dis, 61: 161-166, 2002.

18. Kobayashi A, Naito Houssiau FA, Malaise MG: Increased matrix metalloproteinase- 3 serum levels in rheumatic diseases: relationship with synovitis and steroid treatment. Ann Rheum Dis, 61 : 161-166, 2002.

19. Posthumus MD, Limburg PC, Westra J, Leeuwen MA, Ruswijk MH: Serum matrix metalloproteinase 3 levels during treatment with sulfasalazine or combination of methotrexate and sulfasalazine in patients with early rheumatoid arthritis. J Rheumatol, 29 : 883-889, 2002. 